\title{
Gravitational waves from axionlike particle cosmic string-wall networks
}

\author{
Graciela B. Gelmini ${ }^{*}{ }^{2}$ Anna Simpson $\odot,^{\dagger}$ and Edoardo Vitagliano $\odot^{*}$ \\ Department of Physics and Astronomy, University of California, \\ Los Angeles, California 90095-1547, USA
}

(Received 20 March 2021; accepted 17 August 2021; published 20 September 2021)

\begin{abstract}
Axionlike particles (ALPs) are a compelling candidate for dark matter (DM), whose production is associated with the formation of a string-wall network. If walls bounded by strings persist, which requires the potential to have multiple local minima $(N>1)$, they must annihilate before they become dominant. They annihilate mostly into gravitational waves and nonrelativistic ALPs. We show that for ALPs other than the QCD axion these gravitational waves, if produced at temperatures below $100 \mathrm{eV}$, could be detected by future cosmological probes for ALPs with mass from $10^{-16}$ to $10^{6} \mathrm{eV}$ that could constitute the entirety of the DM.
\end{abstract}

DOI: 10.1103/PhysRevD.104.L061301

\section{INTRODUCTION}

Gravitational waves (GWs) constitute a powerful tool to assess particle physics models [1-5]. They could test in the near future a particular class of light bosonic dark matter (DM) candidates. The production of these particles implies the existence of a stochastic GW background with a peaked spectrum that can be probed by cosmic microwave background $(\mathrm{CMB})$ experiments and astrometry measurements in the $10^{-16} \mathrm{~Hz} \mathrm{GW}-10^{-14} \mathrm{~Hz}$ GW frequency range.

Many extensions of the Standard Model (SM) of elementary particles contain a global $U(1)$ symmetry spontaneously broken at an energy scale $V$ and explicitly broken at another scale $v \ll V$. Models for the original axion [6-8], invisible axions (also called "QCD axions") [9-12], and axionlike particles (ALPs) (e.g., [13-17]) are of this type. In these models, the Nambu-Goldstone (NG) boson corresponding to the spontaneous $U(1)$ breaking acquires a mass $m_{a} \simeq v^{2} / V$, becoming a pseudo-NG boson which we denote with $a$ and call an ALP.

If the spontaneous symmetry breaking happens after inflation, as we assume here, a system of cosmic walls bounded by strings is produced (see e.g., Ref. [18] and references therein). Global cosmic strings are created during the spontaneous symmetry breaking, and become connected by walls at a later time $t \simeq m_{a}^{-1}$. After the explicit breaking, the potential may have just one minimum, $N=1$, or several, $N>1$. With $N=1$, "ribbons" of walls bounded

\footnotetext{
*gelmini@physics.ucla.edu

ansimps@g.ucla.edu

edoardo@physics.ucla.edu
}

Published by the American Physical Society under the terms of the Creative Commons Attribution 4.0 International license. Further distribution of this work must maintain attribution to the author(s) and the published article's title, journal citation, and DOI. Funded by SCOAP. by strings surrounded by true vacuum form, which shrink due to the pull of the walls on the strings. Thus, the wallstring system decays immediately after wall formation, leading to GWs produced only by strings before walls form, observable in future pulsar arrays and direct detection experiments if $V \gtrsim 10^{14} \mathrm{GeV}$ [19].

With $N>1$, each string connects to several walls, forming a stable string-wall system. This system would come to dominate the energy density of the Universe, leading to an unacceptable cosmology unless it disappears early enough [20]. A "bias"- a small energy difference between the vacua at both sides of each wall-would accelerate each wall towards its adjacent higher energy vacuum, driving the domain walls towards their annihilation [20] (see also e.g., Ref. [21]). An additional explicit breaking term in the scalar potential was thus proposed to produce this bias $[22,23]$. This term leads to the existence of one true vacuum, and a bias that we parametrize as $\epsilon_{b} v^{4}$, with a dimensionless positive parameter $\epsilon_{b} \ll 1$.

Gravitational waves due to cosmic strings have been recently studied for NG boson models [24] and $N=1$ ALP models [19]. We focus on models with $N>1$, in which for small enough values of $\epsilon_{b}$, GWs are dominantly produced when the string-wall system annihilates.

\section{ALP MODELS AND THEIR COSMOLOGY}

A generic parametrization for the potential $V(\phi)$ of a pseudo-NG boson model with multiple vacua, and a small bias among them to make the model cosmologically viable (see e.g., [22,23]), includes the terms

$$
\begin{aligned}
V(\phi) \supset & \frac{\lambda}{4}\left(|\phi|^{2}-V^{2}\right)^{2}+\frac{v^{4}}{2}\left(1-\frac{|\phi|}{V} \cos (N \theta)\right) \\
& -\epsilon_{b} v^{4} \frac{|\phi|}{V} \cos (\theta-\delta),
\end{aligned}
$$


where $\phi=|\phi| e^{i \theta}, v \ll V$, and $V \lesssim 10^{16} \mathrm{GeV}$ due to upper bounds on the inflation scale $[25,26]$. The first term is $U(1)$ invariant. It leads to the spontaneous breaking of this symmetry at a temperature $T \simeq V$. Shortly after, $|\phi|=V$, and the phase $\theta=a / V$ has different random values in different patches of the Universe, which leads to the formation of cosmic strings. We assume the bosons have the same temperature or average energy as visible sector particles before the spontaneous breaking, as happens in many inflationary models. In a short time, the Hubble expansion and string recombination lead the string system to a scaling regime, in which the population of strings remains of $\mathcal{O}(1)$ per Hubble volume.

The second term in Eq. (1) breaks $U(1)$ into a $Z_{N}$ discrete subgroup. It produces $N$ degenerate minima with different values of $\theta$, and an ALP mass $m_{a}=v^{2} N /(\sqrt{2} V)$. We assume that $a$ couplings are small enough so that temperature corrections to $m_{a}$ are negligible.

At this point, the equation of motion of the field $a$ in the expanding Universe is that of a harmonic oscillator with damping term $3 H \dot{a}$, where $H=(2 t)^{-1}$ is the Hubble expansion rate during the radiation dominated epoch. At a temperature $T_{w}$ when $H\left(T_{w}\right) \simeq m_{a} / 3$,

$$
T_{w} \simeq \frac{5.1 \times 10^{4} \mathrm{GeV}}{\left[g_{\star}\left(T_{w}\right)\right]^{1 / 4}}\left(\frac{m_{a}}{\mathrm{eV}}\right)^{1 / 2},
$$

regions of the Universe with different values of $\theta$ evolve to different minima and become separated by domain walls of mass per unit area $\sigma=f_{\sigma} v^{2} V / N$. Here $f_{\sigma}$ is a model dependent dimensionless parameter $(\simeq 6$ for $N=2)$. Our figures assume $N=6$ and $f_{\sigma} / N \simeq 1$. In a short time, the expansion of the Universe and energy losses drive the string-wall system into a scaling regime, in which the energy density is $\rho_{w} \simeq \sigma / t$.

The third term in Eq. (1) [22], assumed to be much smaller than the second one, i.e., $\epsilon_{b} \ll 1$, makes the vacuum closest to the arbitrary fixed phase $\delta$ the true one, and raises the others by an energy density difference, a bias, of order $V_{\text {bias }} \simeq \epsilon_{b} v^{4}$. We remain agnostic about the origin of this term (see e.g., Refs. [27-30]).

The surface tension of the walls tends to rapidly straighten out curved walls to the horizon scale $H^{-1}$, and produces a pressure $p_{T} \simeq \sigma / t$, which decreases with time. The volume pressure $p_{V} \simeq V_{\text {bias }}$ tends instead to accelerate the walls towards their lower energy adjacent vacuum, converting the higher energy vacuum into the lower energy one. Assuming that when walls form $p_{V} \ll$ $p_{T}$ (i.e., $\epsilon_{b} \ll 1$ ), at a later time, when $p_{T} \simeq p_{V}$, the bias drives the walls (and the strings bounding them) to annihilate within a Hubble time, when the temperature is

$$
T_{\mathrm{ann}} \simeq \frac{0.73 \times 10^{5} \mathrm{GeV}}{\left[g_{\star}\left(T_{\mathrm{ann}}\right)\right]^{1 / 4}} \sqrt{\frac{\epsilon_{b} m_{a}}{f_{\sigma} \mathrm{eV}}} .
$$

At this point the energy stored in the string-wall system goes entirely into GWs and nonrelativistic or mildly relativistic ALPs (since the wall thickness is $\simeq m_{a}^{-1}$ ) [31].

\section{PRESENT GW ENERGY DENSITY}

The quadrupole formula for the power emitted in GWs $P \simeq G \dddot{Q}_{i j} \dddot{Q}_{i j}$ is used to estimate the GW energy produced by the string-wall system [2]. In the scaling regime the linear size of large walls is $\simeq t$, thus their quadrupole moment as function of the energy in the walls $E_{w} \simeq \sigma t^{2}$ is $Q_{i j} \simeq E_{w} t^{2}$. Thus $\dddot{Q}_{i j} \simeq \sigma t$, and the power emitted in GWs is $P \simeq G \sigma^{2} t^{2}$. The energy density $\Delta \rho_{\mathrm{GW}}$ emitted in a time interval $\Delta t$ is then $\Delta \rho_{\mathrm{GW}}(t) \simeq G \sigma^{2}(\Delta t / t)$. The resulting emitted energy density in a Hubble time $\Delta t=t$ is $\simeq G \sigma^{2}$, independently of the emission time, and for later emission it is less red-shifted. Therefore, the largest contribution to the present GW energy density spectrum, the peak amplitude, corresponds to the time of wall annihilation (a similar calculation can be found e.g., in Ref. [32])

$$
\left.\Omega_{\mathrm{GW}} h^{2}\right|_{\text {peak }} \simeq \frac{1.2 \times 10^{-79} \epsilon_{g w} g_{\star}\left(T_{\mathrm{ann}}\right)}{\epsilon_{b}^{2}\left[g_{s \star}\left(T_{\mathrm{ann}}\right)\right]^{4 / 3}}\left(\frac{f_{\sigma} V}{N \mathrm{GeV}}\right)^{4},
$$

where $\left(g_{\star}\right.$ and $g_{s \star}$ are the energy and entropy density numbers of degrees of freedom), see also Ref. [33]. We include in Eq. (4) a dimensionless factor $\epsilon_{g w}$ found in numerical simulations (see Fig. 8 of Ref. [33]) and conservatively take $\epsilon_{g w}=10$.

Notice that $\Delta \rho_{\mathrm{GW}}(t)$ above defines also the maximum of the GW energy density spectrum at time $t$ as a function of the wave-number at present $k$ (which, when defining the present value of the scale factor $R_{0}=1$, coincides with the comoving wave number) or of the frequency $f=k /(2 \pi)$, which is defined as $\Omega_{\mathrm{GW}} h^{2}(k, t)=\left(h^{2} / \rho_{c}(t)\right)\left(d \rho_{\mathrm{GW}}(t) / d \ln k\right)$, i.e., $d \rho_{\mathrm{GW}}(t) / d \ln (k) \simeq G \sigma^{2}$ (see e.g., Ref. [32]). Thus the peak amplitude of this GW spectrum at present, for $t=t_{0}$, coincides with the total amplitude in Eq. (4).

The peak GW density is emitted at annihilation with frequency $\simeq H\left(T_{\text {ann }}\right)$, which is redshifted to

$$
f_{\text {peak }} \simeq 0.76 \times 10^{-7} \mathrm{~Hz} \frac{T_{\mathrm{ann}}}{\mathrm{GeV}} \frac{\left[g_{\star}\left(T_{\mathrm{ann}}\right)\right]^{1 / 2}}{\left[g_{s \star}\left(T_{\mathrm{ann}}\right)\right]^{1 / 3}} .
$$

The limit $T_{\text {ann }}>5 \mathrm{eV}$ (safely above matter-radiation equality) thus implies $f_{\text {peak }}>5 \times 10^{-16} \mathrm{~Hz}$.

The GW spectrum emitted by cosmic walls for $N>1$ computed numerically is shown in Fig. 6 of Ref. [33]. It has a peak at $f_{\text {peak }} \simeq R\left(t_{f}\right) m_{a}$ and a bump at $f \simeq R\left(t_{f}\right) H\left(t_{f}\right)$, where $t_{f}$ is the latest time in their simulation. Frequencies $f<f_{\text {peak }}$ correspond to super-horizon wavelengths at annihilation, so causality requires a $\sim f^{3}$ dependence [34] for wavelengths that enter into the horizon during 


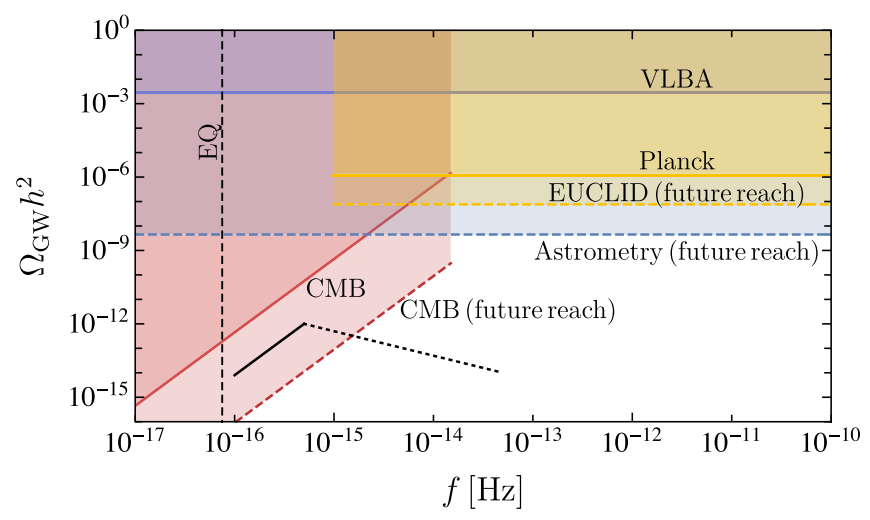

FIG. 1. Regions of $\Omega_{\mathrm{GW}} h^{2}$ vs GW frequency, excluded by existing bounds (solid colored lines) or within the expected reach (dashed colored lines) of $N_{\text {eff }}[39,40]$ (yellow), astrometry [38,41] (blue) and CMB [51] (red) measurements. An example of a differential spectrum from string-wall annihilation is shown, with $T_{\text {ann }}=5 \mathrm{eV}$ and $\left.\Omega_{\mathrm{GW}} h^{2}\right|_{\text {peak }} \simeq 10^{-12}$ : the $\sim f^{3}$ spectrum (solid black line) below the peak is predicted by causality while the $\sim f^{-1}$ spectrum above the peak (dotted black line) is uncertain. The vertical dashed line indicates the frequency of GWs produced at matter-radiation equality.

radiation domination, see e.g., [35-37]. For $f>f_{\text {peak }}$ the spectrum depends instead on the particular production model. Reference [33] finds a $1 / f$ dependence, although the approximate slope and height of the bump depend on $N$.

An example of the approximate spectrum is shown in Fig. 1, together with several present bounds and projected reaches of $\mathrm{GW}$ detection in the near future. For $f>10^{-14} \mathrm{~Hz}$, the most important bounds come from the very long baseline array astrometric catalog [38] (since GWs would produce an apparent distortion of the position of background sources) and from the effective number of neutrino species $N_{\text {eff }}$ during CMB emission [39] (since GWs are a radiation component). EUCLID will improve this latter limit by one order of magnitude [40], and astrometry could reach $\Omega_{\mathrm{GW}} \simeq 10^{-8}$ [41]. At lower frequencies, GWs are constrained by CMB polarization data [42-46]. The present bounds from Planck temperature [26] and BICEP/Keck Array polarization [47] data sets could be improved by planned experiments such as LiteBIRD [48], PICO [49], and CORE [50]. We show the CMB constraints and projections of Ref. [51] for monochromatic GWs, which may be closer to the peaked spectrum of our model than the usually assumed power-law spectrum.

The spectrum of GWs emitted during radiation domination by strings before walls are formed, computed in Refs. $[19,24,52]$, can be approximated with the simple expression

$$
\Omega_{\mathrm{GW}}^{\mathrm{st}} h^{2} \simeq 2 \times 10^{-15}\left(\frac{10^{-12} \mathrm{~Hz}}{f}\right)^{1 / 8}\left(\frac{V}{10^{14} \mathrm{GeV}}\right)^{4} .
$$

This spectrum, very different from the peaked spectrum produced by the string-wall network, does not extend to $f<10^{-12} \mathrm{~Hz}$. In fact, the Ly- $\alpha$ limit on ALP DM $m_{a}>2 \times 10^{-20} \mathrm{eV}$ [53] imposes a limit $T_{w}>5.3 \mathrm{keV}$ (see Eq. (2) which, replacing $T_{\text {ann }}$ by $T_{w}$ in Eq. (5), implies $f>4.7 \times 10^{-11} \mathrm{~Hz}$.

The spectrum cuts off at higher $f$ for larger $m_{a}$ (see Fig. 4 of Ref. [19]). Therefore, in our model the only source of GWs with $f<10^{-12} \mathrm{~Hz}$ is the string-wall system. As clearly shown in Ref. [19] for $N=1$ only a spontaneuos breaking scale $V \gtrsim 10^{14} \mathrm{GeV}$ and $m_{a} \lesssim 10^{-17} \mathrm{GeV}$ can give an observable signal. Thus, for lower breaking scales and heavier ALPs, the only hope to detect GWs associated to ALP production is within the scenario we consider here, with $N>1$.

\section{PRESENT ALP ENERGY DENSITY}

Specifying to our model the analytic derivations in the literature (see e.g., Refs. [33,52,54,55] and references therein) we obtain the different components of the present ALP density.

The ALPs are produced by the string-wall system mostly at annihilation, with average energy $\simeq \sqrt{2} m_{a}$,

$\Omega_{a} h^{2} \simeq \frac{2.4 \times 10^{-24}}{\epsilon_{b}^{1 / 2}}\left(\frac{f_{\sigma}^{3 / 4} V}{N \mathrm{GeV}}\right)^{2}\left(\frac{m_{a}}{\mathrm{eV}}\right)^{1 / 2} \frac{\left[g_{\star}\left(T_{\mathrm{ann}}\right)\right]^{3 / 4}}{g_{s \star}\left(T_{\mathrm{ann}}\right)}$.

Comparing this result with Eq. (19) of Ref. [19], we find that for $\epsilon_{b} \lesssim 2 \times 10^{-9}$ the string-wall ALP production dominates over that of strings, which emit ALPs continuously until walls form. The component of the ALP density due to the initial misalignment of the ALP field is always subdominant. The single contributions to the axion population due to misalignment and string decay are affected

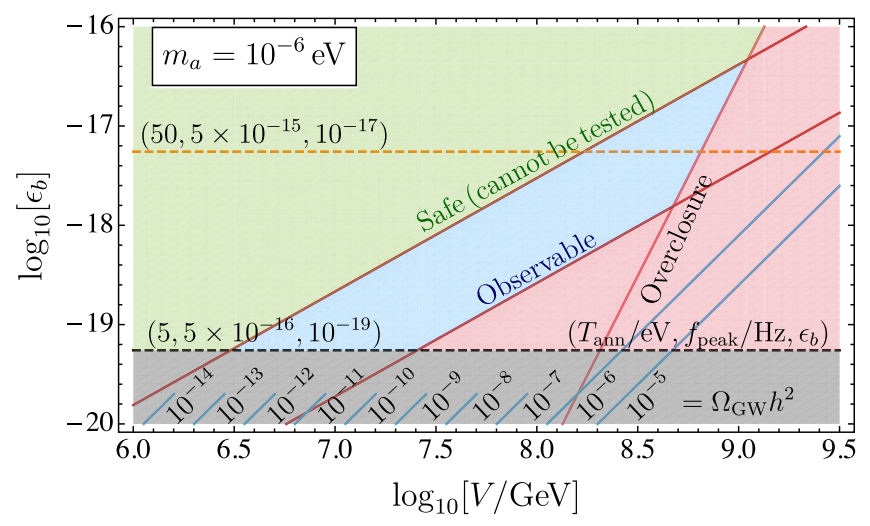

FIG. 2. Regions of interest in $\left\{V, \epsilon_{b}\right\}$ space for $m_{a}=10^{-6} \mathrm{eV}$. Red regions are excluded by either an ALP density larger than that of DM or current CMB limits on GWs in Fig. 1. The gray region corresponds to $T_{\text {ann }}<5 \mathrm{eV}$. The blue region will be explored in the near future by $\mathrm{CMB}$ probes and astrometry. The green region is allowed but not testable. 


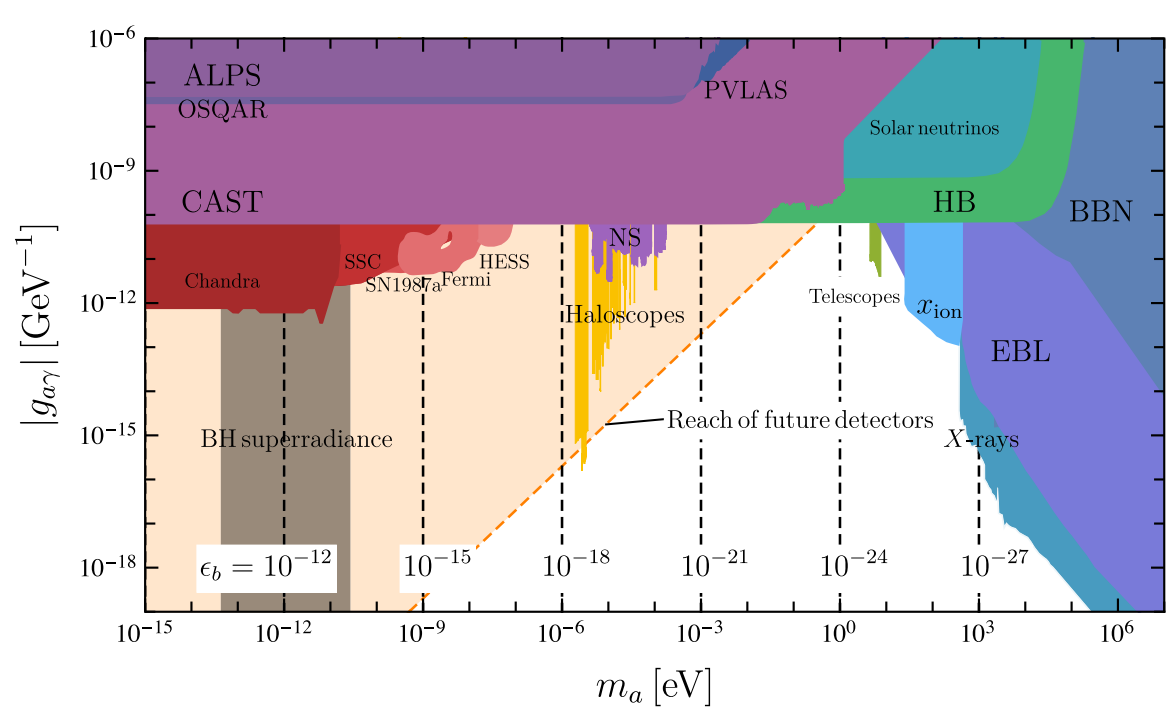

FIG. 3. Parameter space $\left\{m_{a}, g_{a \gamma}\right\}$ for ALPs with coupling $g_{a \gamma} a F \tilde{F}$ to two photons and current bounds from laboratory [66-68], stellar [69,70], other astrophysical [71-76] and cosmological [77-79] measurements, helioscopes [80,81], and direct DM detection [82-92] (see this URL ${ }^{1}$ ). For each $m_{a}$, there is a range of about two orders of magnitude centered at the given $\epsilon_{b}$ in which GWs can be detected. This range is independent of $g_{a \gamma}$, as are the black hole superradiance limits. The latter exclude the brown band [58] (and e.g., Refs. [14,93-98]). The light orange region will be probed by future experiments, e.g., [99-106].

by large uncertainties (see e.g., [56,57]). However, for small enough values of $\epsilon_{b}$ (like those considered in our figures), wall annihilation is in any event the dominant production mechanism.

Combining Eqs. (3)-(5), and (7), the overdensity limit $\Omega_{a} h^{2}<\Omega_{\mathrm{DM}} h^{2}$ implies (neglecting degrees of freedom)

$$
\frac{\left.\Omega_{\mathrm{GW}} h^{2}\right|_{\text {peak }}}{10^{-15}}\left(\frac{f_{\text {peak }}}{10^{-9} \mathrm{~Hz}}\right)^{2}<10^{-4},
$$

which shows that our allowed window is at frequencies below the $10^{-9} \mathrm{~Hz}-10^{3} \mathrm{~Hz}$ range observable in direct GW detection for $\Omega_{\mathrm{GW}} h^{2}>10^{-15}$. For example, for the future reach of astrometry, $\Omega_{\mathrm{GW}} h^{2} \simeq 10^{-9}$, Eq. (8) implies $f_{\text {peak }}<10^{-14} \mathrm{~Hz}$.

\section{GW OBSERVABILITY}

The region of the $\left\{\epsilon_{b}, V\right\}$ space which can be explored by forthcoming measurements of low frequency GWs depends on $m_{a}$. It is shown in blue in Fig. 2 for $m_{a}=$ $10^{-6} \mathrm{eV}$. The GWs are observable for $5 \times 10^{-16} \mathrm{~Hz}<$ $f_{\text {peak }}<1 \times 10^{-14} \mathrm{~Hz}$, i.e., for $5 \mathrm{eV}<T_{\text {ann }}<10^{2} \mathrm{eV}$. Note from Eqs. (3) and (5) that $f_{\text {peak }} \sim \epsilon_{b}^{1 / 2}$, as shown in Fig. 2. The red region is excluded either by ALPs overclosing the Universe or by the current CMB limits shown in Fig. 1, the gray region is excluded because $T_{\text {ann }} \lesssim 5 \mathrm{eV}$, and the green region is allowed but the GW energy density is too small to be detected in the near future. ALPs constitute the whole of the DM on the "Overclosure" line.

\footnotetext{
${ }^{1}$ https://cajohare.github.io/AxionLimits/
}

Combining Eqs. (3)-(5), and (7) one sees that the observable region shifts as $V \sim m_{a}^{-1 / 2}$ and $\epsilon_{b} \sim m_{a}^{-1}$. As $m_{a}$ increases, the lowest $V$ value of the window decreases as $V=10^{6.5} \mathrm{GeV}\left(10^{-6} \mathrm{eV} / m_{a}\right)^{1 / 2}$. Considering the hierarchy of the terms in Eq. (1) we require $v<10^{-2} V$, i.e., $m_{a}<10^{-4} N V$. For $N=6$, this limit restricts the observable window to $V>2.5 \mathrm{GeV}$ and $m_{a}<1.5 \mathrm{MeV}$.

The scaling of the characteristic $\epsilon_{b}$ of the observable window, $\epsilon_{b}=10^{-18}\left(10^{-6} \mathrm{eV} / m_{a}\right)$, shows that ALPs are dominantly produced by walls for $m_{a}>5 \times 10^{-16} \mathrm{eV}$ (for which $\epsilon_{b}<2 \times 10^{-9}$ ). Thus, the observable region in Fig. 2 just translates with the same shape for $m_{a} \gtrsim 10^{-16} \mathrm{eV}$. We do not consider lighter ALPs to avoid black hole superradiance limits, which also reject $3.8 \times 10^{-14} \mathrm{eV}<m_{a}<$ $3.4 \times 10^{-11} \mathrm{eV}$ [58].

Figure 3 shows ALP-photon-photon coupling limits and the characteristic $\epsilon_{b}$ for observable GWs as functions of $m_{a}$. The $\epsilon_{b}$ range of observable $\mathrm{GWs}$ centered at the value shown is about two orders of magnitude wide. As this range depends only on $m_{a}$, it applies to all ALP couplings (e.g., Refs. [59-61]) or "darker" ALPs [62-65].

If future laboratory searches have a signal compatible with a QCD axion, the detection of GWs with the spectrum we described would challenge the attribution of this signal to a QCD axion, since GWs from QCD axion string-wall networks are not detectable [33].

\section{CONCLUSIONS}

We have presented a novel window in to ALP models which takes advantage of the fast progress expected in GW detection, resulting from a so far overlooked mechanism of 
GW production in ALP models. If the ALP potential has several minima, a bias between them is needed to drive the ensuing string-wall system to annihilate early enough to avoid cosmological problems. For the QCD axion, GWs generated by this mechanism are unobservable, but for other ALPs it could produce GWs is a novel frequency range not previously identified for ALP models. We have found that, if walls annihilate at $5 \mathrm{eV} \lesssim T_{\text {ann }} \lesssim 10^{2} \mathrm{eV}$, GWs can potentially be detected by future $\mathrm{CMB}$ probes and astrometry measurements for ALPs with mass from $10^{-16}$ to $10^{6} \mathrm{eV}$, which could constitute all of the DM.

\section{ACKNOWLEDGMENTS}

The work of G. G. and E. V. was supported in part by the U.S. Department of Energy (DOE) Grant No. DESC0009937.
[1] A. Vilenkin and E.P.S. Shellard, Cosmic Strings and Other Topological Defects (Cambridge University Press, Cambridge, England, 2000).

[2] M. Maggiore, Gravitational Waves. Vol. 1: Theory and Experiments, Oxford Master Series in Physics (Oxford University Press, New York, 2007).

[3] M. Maggiore, Gravitational Waves. Vol. 2: Astrophysics and Cosmology (Oxford University Press, New York, 2018).

[4] B. S. Sathyaprakash and B. F. Schutz, Physics, astrophysics and cosmology with gravitational waves, Living Rev. Relativity 12, 2 (2009).

[5] L. Barack et al., Black holes, gravitational waves and fundamental physics: A roadmap, Classical Quantum Gravity 36, 143001 (2019).

[6] R. D. Peccei and H. R. Quinn, CP Conservation in the Presence of Instantons, Phys. Rev. Lett. 38, 1440 (1977).

[7] S. Weinberg, A New Light Boson?, Phys. Rev. Lett. 40, 223 (1978).

[8] F. Wilczek, Problem of Strong $P$ and $T$ Invariance in the Presence of Instantons, Phys. Rev. Lett. 40, 279 (1978).

[9] J.E. Kim, Weak Interaction Singlet and Strong $C P$ Invariance, Phys. Rev. Lett. 43, 103 (1979).

[10] M. A. Shifman, A. I. Vainshtein, and V. I. Zakharov, Can confinement ensure natural $C P$ invariance of strong interactions?, Nucl. Phys. B166, 493 (1980).

[11] M. Dine, W. Fischler, and M. Srednicki, A simple solution to the strong $C P$ problem with a harmless axion, Phys. Lett. 104B, 199 (1981).

[12] A. R. Zhitnitsky, On possible suppression of the axion hadron interactions. (In Russian), Sov. J. Nucl. Phys. 31, 260 (1980), https://inspirehep.net/literature/157263.

[13] P. Svrcek and E. Witten, Axions in string theory, J. High Energy Phys. 06 (2006) 051.

[14] A. Arvanitaki, S. Dimopoulos, S. Dubovsky, N. Kaloper, and J. March-Russell, String axiverse, Phys. Rev. D 81, 123530 (2010).

[15] B. S. Acharya, K. Bobkov, and P. Kumar, An M theory solution to the strong $C P$ problem and constraints on the axiverse, J. High Energy Phys. 11 (2010) 105.

[16] M. Dine, G. Festuccia, J. Kehayias, and W. Wu, Axions in the landscape and string theory, J. High Energy Phys. 01 (2011) 012.
[17] J. Jaeckel and A. Ringwald, The low-energy frontier of particle physics, Annu. Rev. Nucl. Part. Sci. 60, 405 (2010).

[18] A. Vilenkin, Cosmic strings and domain walls, Phys. Rep. 121, 263 (1985).

[19] M. Gorghetto, E. Hardy, and H. Nicolaescu, Observing invisible axions with gravitational waves, J. Cosmol. Astropart. Phys. 06 (2021) 034.

[20] Y. Zeldovich, I. Kobzarev, and L. Okun, Cosmological consequences of a spontaneous breakdown of a discrete symmetry, Zh. Eksp. Teor. Fiz. 67, 3 (1974), https://ui .adsabs.harvard.edu/abs/1975JETP...40....1Z/abstract.

[21] G. B. Gelmini, M. Gleiser, and E. W. Kolb, Cosmology of biased discrete symmetry breaking, Phys. Rev. D 39, 1558 (1989).

[22] P. Sikivie, Of Axions, Domain Walls and the Early Universe, Phys. Rev. Lett. 48, 1156 (1982).

[23] S. Chang, C. Hagmann, and P. Sikivie, Axions from wall decay, Nucl. Phys. B, Proc. Suppl. 72, 99 (1999).

[24] C.-F. Chang and Y. Cui, Stochastic gravitational wave background from global cosmic strings, Phys. Dark Universe 29, 100604 (2020).

[25] M. P. Hertzberg, M. Tegmark, and F. Wilczek, Axion cosmology and the energy scale of inflation, Phys. Rev. D 78, 083507 (2008).

[26] N. Aghanim et al., Planck 2018 results. VI. Cosmological parameters, Astron. Astrophys. 641, A6 (2020).

[27] B. Rai and G. Senjanovic, Gravity and domain wall problem, Phys. Rev. D 49, 2729 (1994).

[28] A. Ringwald and K. Saikawa, Axion dark matter in the post-inflationary Peccei-Quinn symmetry breaking scenario, Phys. Rev. D 93, 085031 (2016); Erratum, Phys. Rev. D 94, 049908 (2016).

[29] F. Ferrer, E. Masso, G. Panico, O. Pujolas, and F. Rompineve, Primordial Black Holes from the QCD Axion, Phys. Rev. Lett. 122, 101301 (2019).

[30] A. Caputo and M. Reig, Cosmic implications of a lowscale solution to the axion domain wall problem, Phys. Rev. D 100, 063530 (2019).

[31] S. Chang, C. Hagmann, and P. Sikivie, Studies of the motion and decay of axion walls bounded by strings, Phys. Rev. D 59, 023505 (1998).

[32] G. B. Gelmini, S. Pascoli, E. Vitagliano, and Y.-L. Zhou, Gravitational wave signatures from discrete flavor symmetries, J. Cosmol. Astropart. Phys. 02 (2021) 032. 
[33] T. Hiramatsu, M. Kawasaki, K. Saikawa, and T. Sekiguchi, Axion cosmology with long-lived domain walls, J. Cosmol. Astropart. Phys. 01 (2013) 001.

[34] C. Caprini, R. Durrer, T. Konstandin, and G. Servant, General properties of the gravitational wave spectrum from phase transitions, Phys. Rev. D 79, 083519 (2009).

[35] G. Barenboim and W.-I. Park, Gravitational waves from first order phase transitions as a probe of an early matter domination era and its inverse problem, Phys. Lett. B 759, 430 (2016).

[36] R.-G. Cai, S. Pi, and M. Sasaki, Universal infrared scaling of gravitational wave background spectra, Phys. Rev. D 102, 083528 (2020).

[37] A. Hook, G. Marques-Tavares, and D. Racco, Causal gravitational waves as a probe of free streaming particles and the expansion of the Universe, J. High Energy Phys. 02 (2021) 117.

[38] J. Darling, A. E. Truebenbach, and J. Paine, Astrometric limits on the stochastic gravitational wave background, Astrophys. J. 861, 113 (2018).

[39] L. Pagano, L. Salvati, and A. Melchiorri, New constraints on primordial gravitational waves from Planck 2015, Phys. Lett. B 760, 823 (2016).

[40] R. Laureijs et al. (EUCLID Collaboration), Euclid definition study report, arXiv:1110.3193.

[41] A. Arvanitaki, S. Dimopoulos, M. Galanis, L. Lehner, J. O. Thompson, and K. Van Tilburg, Large-misalignment mechanism for the formation of compact axion structures: Signatures from the QCD axion to fuzzy dark matter, Phys. Rev. D 101, 083014 (2020).

[42] M. Kamionkowski and A. Kosowsky, The cosmic microwave background and particle physics, Annu. Rev. Nucl. Part. Sci. 49, 77 (1999).

[43] T. L. Smith, M. Kamionkowski, and A. Cooray, Direct detection of the inflationary gravitational wave background, Phys. Rev. D 73, 023504 (2006).

[44] T. J. Clarke, E. J. Copeland, and A. Moss, Constraints on primordial gravitational waves from the Cosmic Microwave Background, J. Cosmol. Astropart. Phys. 10 (2020) 002.

[45] P. D. Lasky et al., Gravitational-Wave Cosmology Across 29 Decades in Frequency, Phys. Rev. X 6, 011035 (2016).

[46] P. Campeti, E. Komatsu, D. Poletti, and C. Baccigalupi, Measuring the spectrum of primordial gravitational waves with CMB, PTA and Laser Interferometers, J. Cosmol. Astropart. Phys. 01 (2021) 012.

[47] P. A. R. Ade et al. (BICEP2 and Keck Array Collaborations), BICEP2/Keck Array x: Constraints on Primordial Gravitational Waves using Planck, WMAP, and New BICEP2/ Keck Observations Through the 2015 Season, Phys. Rev. Lett. 121, 221301 (2018).

[48] T. Matsumura et al., Mission design of LiteBIRD, J. Low Temp. Phys. 176, 733 (2014).

[49] S. Hanany et al. (NASA PICO Collaboration), PICO: Probe of inflation and cosmic origins, arXiv:1902.10541.

[50] J. Delabrouille et al. (CORE Collaboration), Exploring cosmic origins with CORE: Survey requirements and mission design, J. Cosmol. Astropart. Phys. 04 (2018) 014.

[51] T. Namikawa, S. Saga, D. Yamauchi, and A. Taruya, CMB constraints on the stochastic gravitational-wave background at Mpc scales, Phys. Rev. D 100, 021303 (2019).
[52] Y. Gouttenoire, G. Servant, and P. Simakachorn, Beyond the Standard Models with cosmic strings, J. Cosmol. Astropart. Phys. 07 (2020) 032.

[53] K. K. Rogers and H. V. Peiris, Strong Bound on Canonical Ultra-Light Axion Dark Matter from the Lyman-Alpha Forest, Phys. Rev. Lett. 126, 071302 (2021).

[54] T. Hiramatsu, M. Kawasaki, T. Sekiguchi, M. Yamaguchi, and J. Yokoyama, Improved estimation of radiated axions from cosmological axionic strings, Phys. Rev. D 83, 123531 (2011).

[55] M. Gorghetto, E. Hardy, and G. Villadoro, More axions from strings, SciPost Phys. 10, 050 (2021).

[56] M. Gorghetto, E. Hardy, and G. Villadoro, Axions from strings: The attractive solution, J. High Energy Phys. 07 (2018) 151.

[57] V.B. Klaer and G. D. Moore, How to simulate global cosmic strings with large string tension, J. Cosmol. Astropart. Phys. 10 (2017) 043.

[58] M. J. Stott, Ultralight bosonic field mass bounds from astrophysical black hole spin, arXiv:2009.07206.

[59] P. Sikivie, Invisible axion search methods, Rev. Mod. Phys. 93, 015004 (2021).

[60] I. G. Irastorza and J. Redondo, New experimental approaches in the search for axion-like particles, Prog. Part. Nucl. Phys. 102, 89 (2018).

[61] C. A. J. O'Hare and E. Vitagliano, Cornering the axion with $C P$-violating interactions, Phys. Rev. D 102, 115026 (2020).

[62] K. Kaneta, H.-S. Lee, and S. Yun, Portal Connecting Dark Photons and Axions, Phys. Rev. Lett. 118, 101802 (2017).

[63] O. E. Kalashev, A. Kusenko, and E. Vitagliano, Cosmic infrared background excess from axionlike particles and implications for multimessenger observations of blazars, Phys. Rev. D 99, 023002 (2019).

[64] P. Arias, A. Arza, J. Jaeckel, and D. Vargas-Arancibia, Hidden photon dark matter interacting via axion-like particles, J. Cosmol. Astropart. Phys. 05 (2021) 070.

[65] P. deNiverville, H.-S. Lee, and Y.-M. Lee, New searches at the reactor experiments based on the dark axion portal, Phys. Rev. D 103, 075006 (2021).

[66] R. Ballou et al. (OSQAR Collaboration), New exclusion limits on scalar and pseudoscalar axionlike particles from light shining through a wall, Phys. Rev. D 92, 092002 (2015).

[67] F. Della Valle, A. Ejlli, U. Gastaldi, G. Messineo, E. Milotti, R. Pengo, G. Ruoso, and G. Zavattini, The PVLAS experiment: Measuring vacuum magnetic birefringence and dichroism with a birefringent Fabry-Perot cavity, Eur. Phys. J. C 76, 24 (2016).

[68] K. Ehret et al., New ALPS results on hidden-sector lightweights, Phys. Lett. B 689, 149 (2010).

[69] A. Ayala, I. Domínguez, M. Giannotti, A. Mirizzi, and O. Straniero, Revisiting the Bound on Axion-Photon Coupling from Globular Clusters, Phys. Rev. Lett. 113, 191302 (2014).

[70] N. Vinyoles, A. Serenelli, F. L. Villante, S. Basu, J. Redondo, and J. Isern, New axion and hidden photon constraints from a solar data global fit, J. Cosmol. Astropart. Phys. 10 (2015) 015. 
[71] A. Payez, C. Evoli, T. Fischer, M. Giannotti, A. Mirizzi, and A. Ringwald, Revisiting the SN1987A gamma-ray limit on ultralight axion-like particles, J. Cosmol. Astropart. Phys. 02 (2015) 006.

[72] C. S. Reynolds, M. C. D. Marsh, H. R. Russell, A. C. Fabian, R. Smith, F. Tombesi, and S. Veilleux, Astrophysical Limits on Very Light Axion-like Particles from Chandra Grating Spectroscopy of NGC 1275, Astrophys. J. 890, 59 (2020).

[73] C. Dessert, J. W. Foster, and B. R. Safdi, X-ray Searches for Axions from Super Star Clusters, Phys. Rev. Lett. 125, 261102 (2020).

[74] A. Abramowski et al. (H.E.S.S. Collaboration), Constraints on axionlike particles with H.E.S.S. from the irregularity of the PKS 2155-304 energy spectrum, Phys. Rev. D 88, 102003 (2013).

[75] J. W. Foster, Y. Kahn, O. Macias, Z. Sun, R. P. Eatough, V. I. Kondratiev, W. M. Peters, C. Weniger, and B. R. Safdi, Green Bank and Effelsberg Radio Telescope Searches for Axion Dark Matter Conversion in Neutron Star Magnetospheres, Phys. Rev. Lett. 125, 171301 (2020).

[76] M. Ajello et al. (FERMI-LAT Collaboration), Search for Spectral Irregularities due to Photon-Axionlike-Particle Oscillations with the Fermi Large Area Telescope, Phys. Rev. Lett. 116, 161101 (2016).

[77] D. Cadamuro and J. Redondo, Cosmological bounds on pseudo Nambu-Goldstone bosons, J. Cosmol. Astropart. Phys. 02 (2012) 032.

[78] M. Regis, M. Taoso, D. Vaz, J. Brinchmann, S. L. Zoutendijk, N. F. Bouché, and M. Steinmetz, Searching for light in the darkness: Bounds on ALP dark matter with the optical MUSE-faint survey, Phys. Lett. B 814, 136075 (2021).

[79] D. Grin, G. Covone, J.-P. Kneib, M. Kamionkowski, A. Blain, and E. Jullo, A telescope search for decaying relic axions, Phys. Rev. D 75, 105018 (2007).

[80] S. Andriamonje et al. (CAST Collaboration), An Improved limit on the axion-photon coupling from the CAST experiment, J. Cosmol. Astropart. Phys. 04 (2007) 010.

[81] V. Anastassopoulos et al. (CAST Collaboration), New CAST limit on the axion-photon interaction, Nat. Phys. 13, 584 (2017).

[82] S. J. Asztalos et al. (ADMX Collaboration), A SQUIDBased Microwave Cavity Search for Dark-Matter Axions, Phys. Rev. Lett. 104, 041301 (2010).

[83] N. Du et al. (ADMX Collaboration), A Search for Invisible Axion Dark Matter with the Axion Dark Matter Experiment, Phys. Rev. Lett. 120, 151301 (2018).

[84] T. Braine et al. (ADMX Collaboration), Extended Search for the Invisible Axion with the Axion Dark Matter Experiment, Phys. Rev. Lett. 124, 101303 (2020).

[85] C. Boutan et al. (ADMX Collaboration), Piezoelectrically Tuned Multimode Cavity Search for Axion Dark Matter, Phys. Rev. Lett. 121, 261302 (2018).

[86] S. Lee, S. Ahn, J. Choi, B. R. Ko, and Y. K. Semertzidis, Axion Dark Matter Search Around 6.7 $\mu \mathrm{eV}$, Phys. Rev. Lett. 124, 101802 (2020).

[87] L. Zhong et al. (HAYSTAC Collaboration), Results from phase 1 of the HAYSTAC microwave cavity axion experiment, Phys. Rev. D 97, 092001 (2018).
[88] K. M. Backes et al. (HAYSTAC Collaboration), A quantumenhanced search for dark matter axions, Nature (London) 590, 238 (2021).

[89] C. Hagmann, P. Sikivie, N. S. Sullivan, and D. B. Tanner, Results from a search for cosmic axions, Phys. Rev. D 42, 1297 (1990).

[90] B. T. McAllister, G. Flower, E. N. Ivanov, M. Goryachev, J. Bourhill, and M. E. Tobar, The ORGAN Experiment: An axion haloscope above $15 \mathrm{GHz}$, Phys. Dark Universe 18, 67 (2017).

[91] D. Alesini et al., Galactic axions search with a superconducting resonant cavity, Phys. Rev. D 99, 101101 (2019).

[92] J. Schütte-Engel, D. J. E. Marsh, A. J. Millar, A. Sekine, F. Chadha-Day, S. Hoof, M. Ali, K.-C. Fong, E. Hardy, and L. Šmejkal, Axion quasiparticles for axion dark matter detection, arXiv:2102.05366.

[93] A. Arvanitaki and S. Dubovsky, Exploring the string axiverse with precision black hole physics, Phys. Rev. D 83, 044026 (2011).

[94] R. Brito, V. Cardoso, and P. Pani, Superradiance: New frontiers in black hole physics, Lect. Notes Phys. 906, 1 (2015).

[95] T. Ikeda, R. Brito, and V. Cardoso, Blasts of Light from Axions, Phys. Rev. Lett. 122, 081101 (2019).

[96] M. Baryakhtar, M. Galanis, R. Lasenby, and O. Simon, Black hole superradiance of self-interacting scalar fields, Phys. Rev. D 103, 095019 (2021).

[97] D. Blas and S. J. Witte, Imprints of axion superradiance in the CMB, Phys. Rev. D 102, 103018 (2020).

[98] H. Fukuda and K. Nakayama, Aspects of nonlinear effect on black hole superradiance, J. High Energy Phys. 01 (2020) 128.

[99] J. L. Ouellet et al., First Results from ABRACADABRA$10 \mathrm{~cm}$ : A Search for Sub- $\mu \mathrm{eV}$ Axion Dark Matter, Phys. Rev. Lett. 122, 121802 (2019).

[100] I. Shilon, A. Dudarev, H. Silva, and H. H. J. ten Kate, Conceptual design of a new large superconducting toroid for IAXO, the New International AXion Observatory, IEEE Trans. Appl. Supercond. 23, 4500604 (2013).

[101] D. J. E. Marsh, K.-C. Fong, E. W. Lentz, L. Smejkal, and M. N. Ali, Proposal to Detect Dark Matter Using Axionic Topological Antiferromagnets, Phys. Rev. Lett. 123, 121601 (2019).

[102] M. Lawson, A. J. Millar, M. Pancaldi, E. Vitagliano, and F. Wilczek, Tunable Axion Plasma Haloscopes, Phys. Rev. Lett. 123, 141802 (2019).

[103] A. Caldwell, G. Dvali, B. Majorovits, A. Millar, G. Raffelt, J. Redondo, O. Reimann, F. Simon, and F. Steffen (MADMAX Working Group collaboration), Dielectric Haloscopes: A New Way to Detect Axion Dark Matter, Phys. Rev. Lett. 118, 091801 (2017).

[104] M. Baryakhtar, J. Huang, and R. Lasenby, Axion and hidden photon dark matter detection with multilayer optical haloscopes, Phys. Rev. D 98, 035006 (2018).

[105] I. Stern, ADMX status, Proc. Sci., ICHEP2016 (2016) 198 [arXiv:1612.08296].

[106] D. Alesini, D. Babusci, D. Di Gioacchino, C. Gatti, G. Lamanna, and C. Ligi, The KLASH proposal, arXiv: 1707.06010. 